\title{
De-Noising of Uterus Fibroid Ultrasound Image Using Gaussismooth Convolution Filter (GSCF)
}

\author{
Dr. M. Renuka Devi*, V. Sindhu** \\ *Head Department of Computer Applications, \\ Sri Krishna College of Arts and Science, Coimbatore \\ **Research Scholar Bharathiar University, Coimbatore
}

\begin{abstract}
This paper discusses the methods to detect the presence of uterus fibroid in woman by implementing various image processing techniques. The input image is an ultrasound image as it is cost effective when compared to other imaging techniques like CT, MRI. The initial step in image processing is to remove noise by applying filters. Application of filters smoothen the image without blurring the image. Gradient of the processed image is calculated and the image is enhanced by sharpening the edges of the image are achieved by calculating the local maxima of the gradient. Then, the edges are decided by calculating the threshold value of the processed image. The proposed Gaussismooth Convolution Filter gives better results when compared with other existing filter with PSNR value of $94 \%$.
\end{abstract} Keywords:- Uterus, Fibroid, Ultrasound, Gaussismooth
Convolution Filter.

\section{INTRODUCTION}

Nowadays researchers of various domains have gathered interest towards image processing and data mining due its increased efficiency in giving solution to all the problems. Applications of these techniques are cross domain. Digital images are the source of input image processing techniques, which helps in analyzing the images and gives the desired information. Data mining on the other hand plays a vital role in extracting the intended data from the large repository of data. Almost every individual is exercising the benefits of these applications in routine life. Having a study at the application of these techniques resulted in almost most of the fields likes plant disease identification, quality analysis in the finished products in industry, population growth prediction, medical field, etc., though accuracy and reliability of the generated system highly relay on the image quality taken for analysis. Mostly, the quality of the image is altered by the noise that are present the image. Hence it becomes primary step to avoid noise in the image.

\section{$>$ Uterine Fibroid:}

Uterine fibroid is the most common muscle tumor that is found in women of childbearing age i.e from 25 to 40 . Fibroids are mostly harmless. These tumors are categorized into benign tumor which is non-cancerous tumors. These tumors are mostly regenerative in nature. Uterus fibroids can be simply understood as abnormal muscle growth in walls of uterus. These lesions can either single or multiple in number. The lumps can be found in various sizes ranging from $\mathrm{mm}$ to $\mathrm{cm}$. Women with uterus fibroids can be diagnosed with different symptoms like pain in abdomen, patient becoming anemic, heavy menstrual flow and may also experience pain during intercourse based on the location of fibroids in uterus. Some may not have any symptoms. Though these tumors are harmless, in few cases it may turn to malignant (cancer).

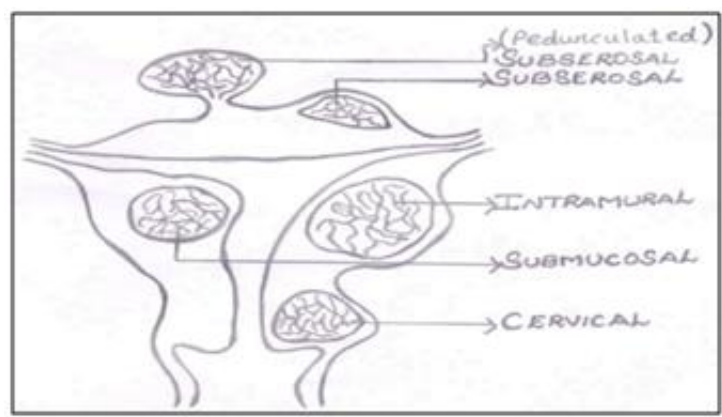

Fig 1:- Types of Uterus Fibroid

Uterus fibroids are categorized into five types based on their region of growth. They are Subserosal (Pedunculated), Submucosal, cervical, and Intramural. Fibroids are diagnosed in many ways like x-rays, ultrasonography, MRI, Hysterosalpingography, Hysteroscopy, Endometrial biopsy, Blood test. Among these techniques ultrasound is the most commonly used method to diagnose the tumor. Ultrasound imaging techniques are very effective due its cost effectiveness and less to zero side effects.

Fibroids are of five types which are categorized based on the location of the fibroid in uterus. Presence of fibroid are diagnosed by using any of the following procedures like $\mathrm{X}^{-}$, rays transvaginal ultrasound which is commonly known as ultrasonography, MRI, Hysterosalpingography, Hysteroscopy, Endometrial biopsy, Blood test. Among all these techniques of diagnosing fibroid presence Ultrasound imaging is the most common and convenient method. Though ultrasound imaging is an effective method in diagnosing fibroids still it suffers from certain setback which hardens decision process for radiologists. In such cases, Data mining techniques provides effective results to overrule the challenges from ultrasound images and to diagnose the disease easily and to provide better healthcare support for the patient. The current research has taken initiative to provide soft computational methods using new data mining and image processing techniques to diagnose the presence of fibroid and classify them based on their type. 


\section{$>$ Problem Definition:}

Nearly $50 \%$ of Women in childbearing age are affected by uterus fibroids. Also another report says that about $70 \%$ of women in age group of 50 are also affected. It witnessed that excesses or insufficient secretion of estrogen and progesterone may lead to growth of uterus fibroids. However the causes of the uterine fibroids are not known clearly. Ultrasound imaging can be used to various soft tissues in body like liver, kidney, uterus, abdomen etc., and for any abnormalities in these soft tissue organs.

Irrespective of the advantages of ultrasound scan it has a greater drawback of having speckle noise in the image. The speckle noise is multiplicative in nature. These are spread throughout the image. Speckle noise eventually decreases the quality of image by degrading the spatial and contrast resolution of the image. This noise makes it difficult for the radiologist to decide about the disease. To address this aspect, the current research has proposed a system aided solution by implying image processing and data mining techniques which provides a supporting hand to the radiologist to improve the diagnostic results.

\section{RESEARCH METHODOLOGY}

The following chart shows the brief flow of research. The current research work is carried on in five phases, each phase is proposed with a new procedure to perform image processing.

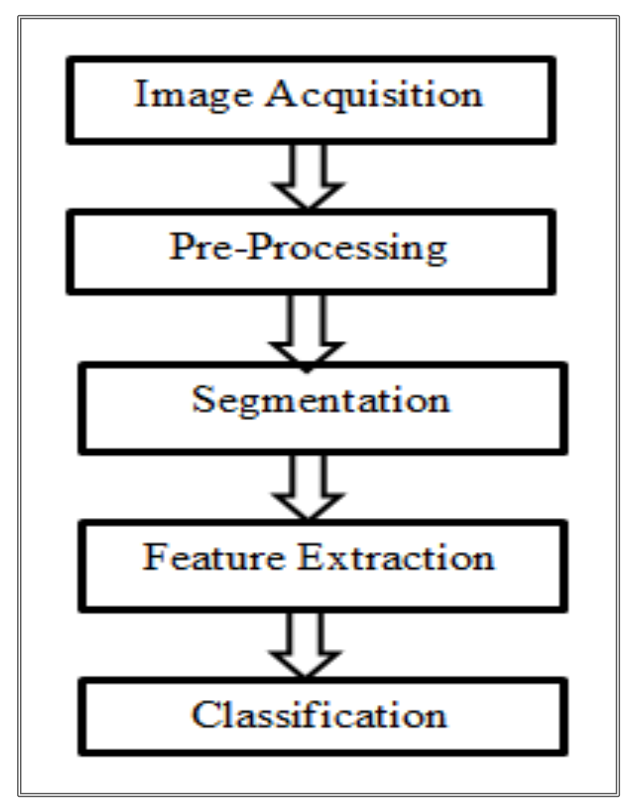

Fig 2:- General Research Flow

\section{> Types of Filters:}

Pre-processing is the initial step in image processing. Pre-Preprocessing of digital image includes noise removal, edge detection, image re-sizing, compressing, etc., These are chosen by the researcher based on the image and requirement of the problem definition. There are number of methods proposed on each category by the researchers' worldwide. The following are the different types of filters that are commonly used in noise removing.

\section{- Mean Filter:}

The working factor of mean filter is to find the mean (average) and replace the pixel value of the image by its neighbor and by including itself. The pixel values that are not represented by its surrounding pixels are eliminated by the mean filter. Mean filter is also referred as Convolution filter. Calculation of mean is done based on the shape and size of the neighborhood pixel sample. The working of mean filter is around the kernel.

\section{- Median Filter:}

The mean filter calculates the mean by considering the pixels that are represented by the neighborhood pixels, the pixels that are not represented by the neighborhood pixels are eliminated. Whereas the median filter in turn looks or searches for the pixel that are represented by neighborhood pixel in the image. It replaces the pixels by median value of those pixels.

\section{- Gaussian Filter:}

The images can be pre-processed using convolution filter which at times blurs the image. Instead the image can be smoothened by applying the Gaussian filter without affecting the original image. Gaussian similar to the mean filter but the kernel chose is different from the mean filter. The kernel that is used by the Gaussian filter is 'bellshaped' hump. It is known as Gaussian hump. The computation behind Gaussian filter is show below:

$$
G(x)=\frac{1}{\sqrt{2 \pi} \sigma} e^{-\frac{x^{2}}{2 \sigma^{2}}}
$$

\section{- Adaptive Filtering}

Adaptive filter is applicable on the images that are corrupted by noise. It is based on the $\mathrm{M} \mathrm{X} \mathrm{N}$ window region of the image. Mean and variance are used in the noise filtering technique. Adaptive filter sounds to be much better when compared to other filters due its ability to preserve the edges and other high-frequency region of the images.

\section{- Wiener Filter:}

Weiner filter uses statistical methods to remove the noise from the image. To apply this filter one should have the spectral properties of the original image. This filter removes the mean square error of the image between the random process and desired process.

\section{- Existing Methods:}

Rundo, L., et al (2019)[1] The author has proposed a novel method to improve the quality of the image that are taken for processing. The proposed method MedGA is a genetic based algorithm which works based on the intensity of the greyscale level of the image. It enhances the histogram level of the image. The performance of the proposed system sound much effective when compared other image enhancement techniques like histogram equalization, decoding and encoding Gamma transformations. 
ISSN No:-2456-2165

Rundo, L., Militello, C., Vitabile, S., Casarino, C., Russo, G., Midiri, M., \& Gilardi, M. C. (2016).[2] this researcher brings an efficient method to detect the presence of fibroids in human using the proposed method, an advanced direct region detection model. This method helps in segmenting the affected region without committing any cause to other regions in uterus. the autjor has also proposed a split and merge technique to select the seeded region. The seed region selection is based on the adaptive region growing technique. The proposed method shows good results with sensitivity of $84.05 \%$, a specificity of $92.84 \%$.

Yang, J., Fan, J., Ai, D., Wang, X., Zheng, Y., Tang, S., \& Wang, Y. (2016). [3] the author proposed an improved method to reduce the presence of speckle noise in the medical image. To reduce the speckle noise in the image author has involved non-local mean filter with redundancy information. the performance of proposed hybrid NLM filter shows better results with the real images and the simulated images.

Sarode, M. V., \& Deshmukh, P. R. (2011). [4] the author has proposed an effective method to reduce the speckle noise present in the image. The poposed method is tested on both the ultrasound image and also on the spatial images. The ultrasound images showed better results by enhance the image quality.

\section{Proposed Methodology:}

Noises in the image are accumulated during the image acquisition stage. Presence of noise in the image affects the pixels of image, resulting in deterioration of image quality. In order to improve the quality of the image it is essential to remove the noises that are present. Images may get affected by various noises such as speckle noise, impulse noise, Gaussian noise and fractal Noise; the foremost task of a researcher is to task of remove these noises in order to accomplish a quality image. It can be attained by implementing various techniques that are available to remove or to limit the presence of noise in the image. Selection of noise removal technique has to be done carefully without affecting the quality of the original image. The current paper concentrates in enhancing accuracy of the noise removal techniques without affecting or blurring the original image. It also increases the PSNR value of the filter.

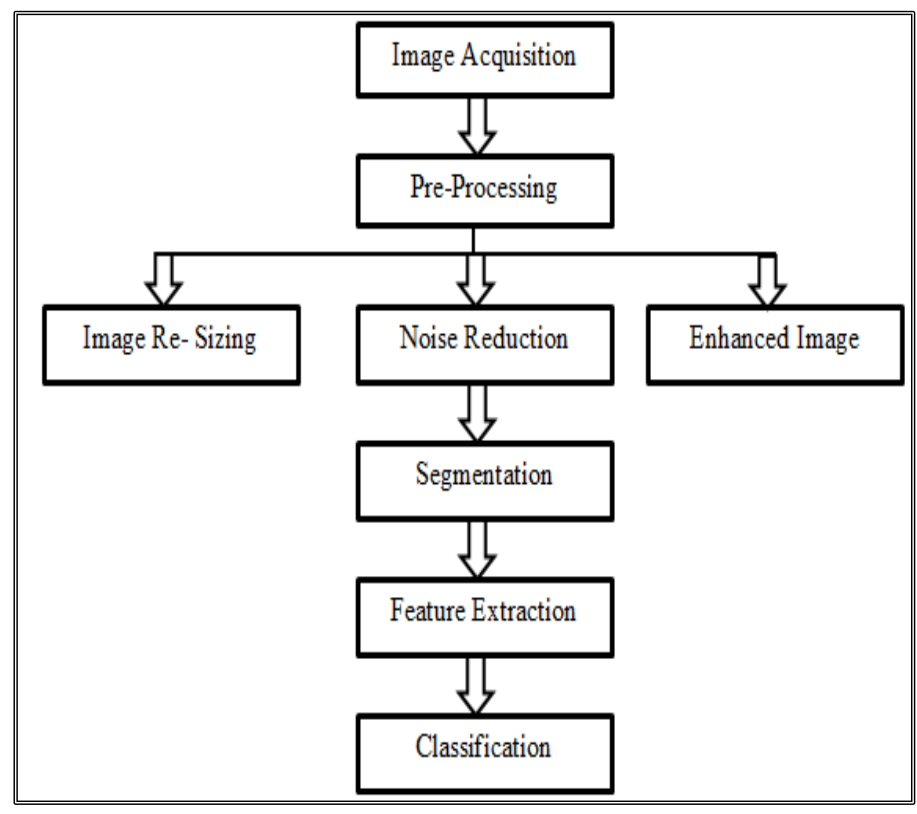

Fig 3:- Steps in Image Pre-Processing

Steps in Proposed Method: Algorithm: Gaussismooth convolution Filter

Step 1: Input: Read the noisy (ULTRASOUND Scan) image

Step 2. Image Resizing: Original Image of size 480 x640 is resized to $256 \times 256$ pixels by $\mathrm{Bi}$ cubic interpolation method.

Step 3. Polishing: Gaussian filter is applied with mask of $3 \times 3$ to remove the noise. Then examine from the $1^{\text {st }}$ pixel and move window towards the last pixels by row and column wise by convoluting the pixels.

$\%$ Indexing Row of noisy pixel

For $\mathrm{r}=(1+\mathrm{P})$ :(row-P)

$\%$ Indexing Column of noisy pixel

for $\mathrm{s}=(1+\mathrm{P})$ : $(\mathrm{clm}-\mathrm{P})$

$\%$ Remove Noise from Image

pixR=nim $(r, s) ; \%$ nim= noisy image

Step 4: Gradient Detection: Gradients and amplitude of the filtered image is calculated by using

$S(a, b)=\mathrm{a}=\sigma^{2} \mathrm{a}, \mathrm{b}+\mathrm{b}=\sigma^{2}(\mathrm{a}, \mathrm{b})$

Step 5: Sharpening: This step aims to sharp the edges of blurred edges. Essentially this is done by continuing all local maxima in the gradient image, and deleting everything else.

Step 6: Binary Thresholding: Edges are decided based on their threshold values. If threshold of Edge pixels are high then it fixed as strong. Else it is negligible.

Step 7: Edge tracking: continue the step 5 for whole image until fix the edges. 
ISSN No:-2456-2165

\section{RESULT AND DISCUSSION}

The performance of the proposed filter was examined by passing ultrasound images of uterus as input. The proposed filter removes the noise. It also enhances the image quality by retaining the edges of the ultrasound image. Gradient of the filtered image is calculated by having a count on the change in the intensity value of the filtered image. Also, amplitude of the pixels is measured. The edges of the images are retained by performing thresholding.

The proposed filter preserves the edges without any loss during the entire process of filter. The performance of the filter is measured using two metrics such as Mean Square Error (MSE) and Peak Signal to Noise (PSNR). The proposed filter is faster in its computational speed.

The proposed GSC filter is implemented by calculating mask. As the mask is calculated the noise in the image is removed by performing double convolution method. The mask which is chosen for convolution is of size $3 \times 3$.

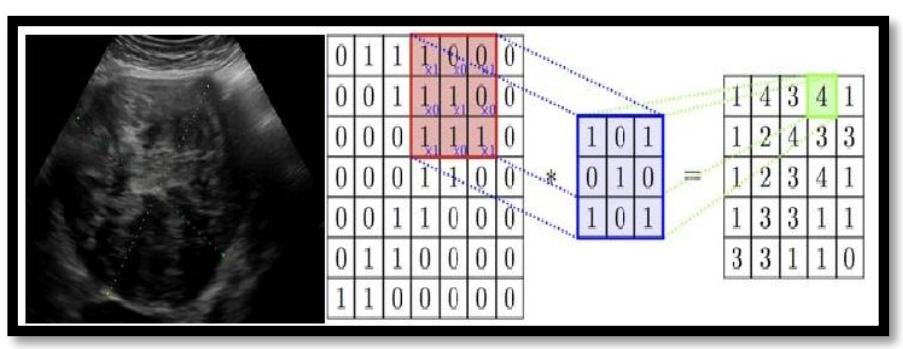

Fig 4:- Convolution in proposed filter

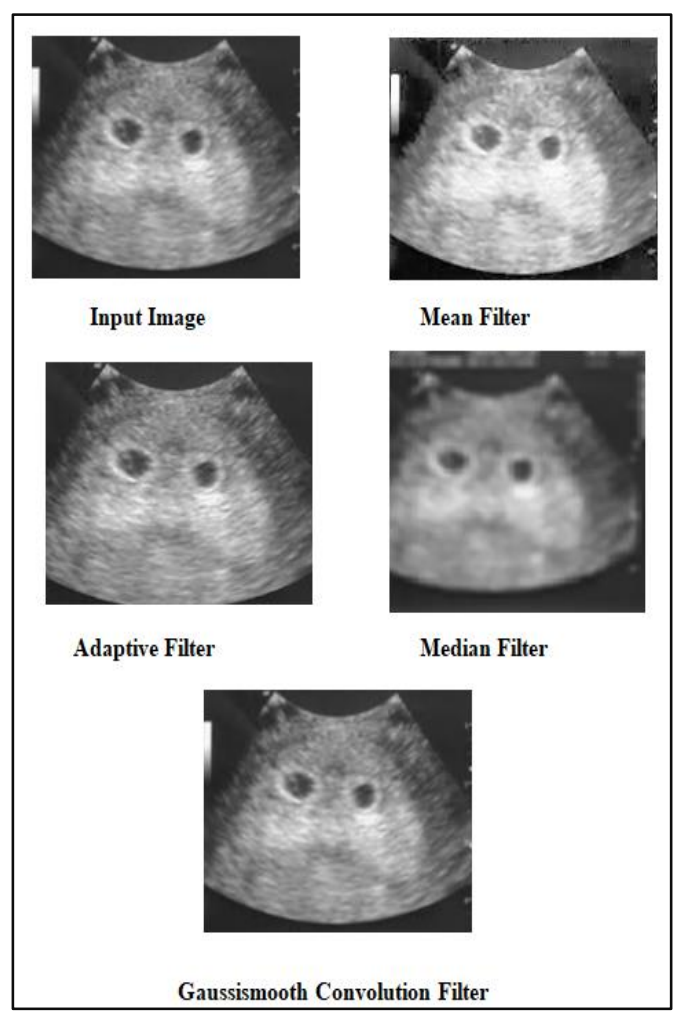

Fig 5:- Results of Various Filters \begin{tabular}{|c|}
\hline$P S N R=10 \log _{10} \frac{255}{R M S E}$ \\
\hline Comparison of PSNR Value of GSCF with Existing Filters
\end{tabular}

The following shows the comparison chart of the various filters with their PSNR value for the input ultrasound uterus image. The proposed GSC filter is compared with mean, median and adaptive filters. The highest PSNR value for the uterus image is given by GSC filter with 94.145 for the uterusimg4.jpg. PSNR value of the image is calculated by the following formula.

\begin{tabular}{|c|c|c|c|c|}
\hline Image & $\begin{array}{c}\text { Mean } \\
\text { Filter }\end{array}$ & $\begin{array}{c}\text { Median } \\
\text { Filter }\end{array}$ & $\begin{array}{c}\text { Adaptive } \\
\text { Filter }\end{array}$ & $\begin{array}{c}\text { GSC } \\
\text { Filter }\end{array}$ \\
\hline UterusImgl.jpg & 84.785 & 75.455 & 62.914 & $\mathbf{9 0 . 1 8 4}$ \\
\hline UterusImg2.jpg & 83.205 & 74.821 & 63.682 & $\mathbf{8 8 . 4 3 7}$ \\
\hline UterusImg3.jpg & 86.336 & 74.963 & 65.566 & $\mathbf{9 4 . 1 4 5}$ \\
\hline UterusImg4.jpg & 89.149 & 78.787 & 68.887 & $\mathbf{9 1 . 3 8 5}$ \\
\hline UterusImg5.jpg & 81.657 & 72.356 & 69.325 & $\mathbf{8 9 . 0 1 7}$ \\
\hline
\end{tabular}

Table 1:- Comparison MSE Value of GSCF with Existing Filters

In Table 1 MSE value of proposed filter is compared with mean, median, Adaptive filters. Among those filters the proposed shows minimum error value when compared with the other existing filters.

\section{CONCLUSION}

The current research work has proposed a new filter Gaussismooth Convolution Filter (GSCF) for removing noise in the ultrasound image of uterus fibroid. The research has also discussed about various other existing filter for noise removal. The proposed filter removes the noise in the image by retaining the edges of the image. The proposed method shows high PSNR value as 94\%. This stands as evidence for the proposed filter having improved results when compared with other filters like Mean, Median and Adaptive. The MSE value of proposed filter is 0.0001 than other filters. Hence, the performance of the proposed Gaussismooth convolution filter is good when compared to other existing filter methods.

\section{REFERENCES}

[1]. Rundo, L., Tangherloni, A., Nobile, M. S., Militello, C., Besozzi, D., Mauri, G., \& Cazzaniga, P. (2019). MedGA: a novel evolutionary method for image enhancement in medical imaging systems. Expert Systems with Applications, 119, 387-399.

[2]. Rundo, L., Militello, C., Vitabile, S., Casarino, C., Russo, G., Midiri, M., \& Gilardi, M. C. (2016). Combining split-and-merge and multi-seed region growing algorithms for uterine fibroid segmentation in 
MRgFUS treatments. Medical \& biological engineering \& computing, 54(7), 1071-1084.

[3]. Yang, J., Fan, J., Ai, D., Wang, X., Zheng, Y., Tang, S., \& Wang, Y. (2016). Local statistics and non-local mean filter for speckle noise reduction in medical ultrasound image. Neurocomputing, 195, 88-95.

[4]. Sarode, M. V., \& Deshmukh, P. R. (2011). Reduction of speckle noise and image enhancement of images using filtering technique. International Journal of Advancements in Technology, 2(1), 30-38.

[5]. Jeyalakshmi, T. R., \& Ramar, K. (2010). A modified method for speckle noise removal in ultrasound medical images. International Journal of Computer and Electrical Engineering, 2(1), 54.

[6]. Renuka Devi. M, V. Sindhu. (2015). A analysis on various data mining approaches to predict fibroid tumor presence in women. Applied Engineering Research ISSN 0973-4562 Volume 10, Number 12 (2015) pp. 28349-28355 